radical mastoidectomy: 47 ; otitis externa: 17 ; perforated tympanic membrane: 8 ; cortical mastoidectomy: 7; myringoplasty: 4 ; fenestration cavity: 2 ; attic retraction pocket: 1 ; T-tube grommet: 1 . Seventy-two of these patients were transferred from the ENT outpatient clinic and as a consequence it is estimated that 252 hospital episodes/year have been saved. Nine patients were referred to C.H.L. by other general practitioner colleagues usually for treatment of long-standing otitis externa. All patients were examined under the microscope in the community clinic but this may not be possible in a busy hospital outpatient clinic where the operating microscope is not always readily available.

All patients in the original pilot project were from C.H.L.s' general practice. Fifty-nine out of eightyseven patients currently under community follow-up are registered with neighbouring practices. Agreement was reached by C.H.L. and A.R. to treat patients from these practices after discussing the project with the Local Medical Committee and also by writing to all local practitioners. No problems have been experienced treating these patients. If rereferral back to the hospital ENT outpatient clinic is required this is performed by C.H.L. and the patient's own general practitioner is notified.

Patients report satisfaction with the community service as appointment times and days are flexible to accommodate patient preference. The average time saved per consultation on attending the community clinic is 86 minutes when travelling, parking and outpatient waiting are taken into account. The microsuction patients are generally seen in C.H.L.s' ordinary surgery and on average can expect to be seen within five minutes of their appointment time.

In our paper we were unable to define the ideal population size to support a community clinic. We felt that eventually 250 patients' care might be transferred but this figure may be over optimistic. At present 87 patients are being treated and this figure continues to slowly increase.

Undoubtedly routine mastoid examination and microsuction can be performed in a variety of clinical settings. The North Staffordshire model using a specially trained nurse will be appropriate in some areas and our community-based service suitable elsewhere. Hospital-based services, however, do generally suffer from the disadvantages of inflexible appointment times, problems with travelling to and parking at the hospital and treatment by different staff at each attendance without the continuity of care and level of expertise offered by the Portsmouth Project.

Adel Resouly, F.R.C.S., Charles Lewis, M.D.

\section{Analysis of CT scanning referrals for chronic rhinosinusitis}

Dear Sir,

I read with great interest the article by White et al. in the Journal of Laryngology and Otology in July 1996, entitled 'Analysis of CT scanning referrals for chronic rhinosinusitis'. The authors have correctly emphasized the importance of prior medical therapy and nasal endoscopy before scanning. The potential side effects of radiation are also rightly emphasized.

However, the authors' conviction that use of CT 'purely as a diagnostic test for rhinosinusitis is inappropriate' does not appear to be well founded. In clinical practice, sometimes in spite of a thorough history, physical examination and nasal endoscopy diagnosis of chronic rhinosinusitis can not be confirmed or ruled out. Clinicians, then have to rely on radiologic means to make a diagnosis. The fallacy of plain sinus radiographs in these situations has been well described (Mafee, 1994). The usefulness of CT scan in these situations cannot be overemphasized. In these patients, at least a limited sinus CT scan may be considered. The dosage of radiation in the studies have been described to be acceptable (White et al., 1991; Rowe-Jones et al., 1995). From a clinicians viewpoint, its unfair to describe sinus CT as purely pre-operative or to expect about 80 per cent of those having CT scans to go for surgery.

\section{References}

Mafee, M. F. (1994) Modern imaging of paranasal sinuses and the role of limited sinus computerized tomography: consideration of time, cost and radiation. Ear, Nose and Throat 73: 532-546.

Rowe-Jones, J., MacKay, I., Colquhoun, I. (1995) Charing Cross CT Protocol for endoscopic sinus surgery. The Journal of Laryngology and Otology 109: 1057-1060.

White, P. S., Cowan, I. A., Robertson, M. S. (1991) Limited sinus CT scanning techniques of the paranasal sinuses. The Journal of Laryngology and Otology 105: 20-23.

\section{Author's reply}

Your reader highlights an important issue. This audit of referral practice was instigated at the request of our radiology colleagues who had expressed concern over the number of otherwise healthy patients undergoing CT scanning. I take issue with the view that 'the dosage of radiation. . . have been described to be acceptable', noting that the references quoted predate Maclennan, (Maclennan, 1995). He measured the tissue radiation dose associated with sinus CT, (including mini-scan protocols) throughout Scotland, and demonstrated radiation doses of between three and 14 times the annual average recommended maximum dose to the general public. The investigation then is clearly not without some risk, and while we are not suggesting the CT cannot be used as a diagnostic tool for rhinosinusitis, its use should be limited. Our pre-set standard of 80 per cent of scanned patients reflected the view of our panel of experts, that the scan should be used primarily for planning surgery. This does not mean that in a small minority of patients where diagnosis is proving difficult, despite a full clinical assessment and nasendoscopy, that a CT scan should not be performed. As we stated we accept that a standard of good practice could be established arbitarily below the 80 per cent level, and this a matter for local 
agreement between radiologist and surgeon. It is important that CT not be seen as the new replacement for sinus $\mathrm{X}$-rays, otherwise the number of patients being subjected to the radiation exposure associated with CT scanning unnecessarily will continue to rise.

Mr P. S.White,

Consultant Otolaryngologist,
Ninewells Hospital and Medical School, Dundee DD1 9SY.

\section{Reference}

Maclennan, A. C. (1995) Radiation dose to the lens from coronal CT scanning of the sinuses. Clinical Radiology 50: 986-988. 